\title{
Aspectos neurorradiológicos do transtorno obsessivo-compulsivo: o papel dos gânglios da base
}

\author{
Antonio Augusto Valente J r. e Geraldo Busatto Filho
}

\begin{abstract}
Departamento e Instituto de Psiquiatria e Centro de Medicina Nuclear da Faculdade de Medicina da USP. São Paulo, SP, Brasil
\end{abstract}
\begin{abstract}
Contemporary neuroanatomical models for obsessive-compulsive disorder (OCD) propose that abnormalities of specific cortico-subcortical circuits mediate the disorder's symptoms. This paper focuses on the basal ganglia, believed to play a critical role in the functioning of those brain circuits. Clinical and experimental studies investigating the presence of abnormalities of basal ganglia structures in OCD are reviewed, with particular emphasis on the neuroimaging literature. Despite controversies in the area, a considerable amount of data has accumulated suggesting that abnormalities of the caudate and putamen nuclei can be associated with OCD. These abnormalities may be particularly relevant to specific subtypes of the disorder, such as early-onset OCD. With the continuing sophistication of neuroimaging technologies, future studies may be able to further clarify the role of each basal ganglia component in the pathophysiology of the various clinical features of OCD.
\end{abstract}

Keywords Obsessive-compulsive disorder. Neuroanatomy. Diagnostic imaging. Basal ganglia.

\section{Introdução}

Apesar dos avanços no conhecimento clínico sobre o transtorno obsessivo-compulsivo (TOC) e da disponibilidade de tratamentos eficazes, as causas e a fisiopatologia desse transtorno ainda necessitam ser melhor esclarecidas.

Modelos neuroanatômicos têm sido propostos para o TOC, enfatizando o papel de circuitos neuronais que interconectam regiões específicas do córtex cerebral e estruturas subcorticais. Esses modelos recebem suporte de pesquisas utilizando técnicas de neuroimagem, que mostram, consistentemente, alterações estruturais e funcionais envolvendo córtex órbito-frontal, cíngulo anterior, gânglios da base e tálamo em grupos de pacientes com TOC comparados a controles normais. ${ }^{1}$

No presente artigo, abordar-se-á especificamente o papel dos gânglios da base (GB), estruturas de importância crítica dentro dos circuitos córtico-subcorticais envolvidos na fisiopatologia do TOC.

Os GB constituem um conjunto de massas de substância cinzenta subcorticais interconectadas, incluindo núcleo caudado e putâmen (que juntos formam o corpo estriado), globo pálido, substância negra e núcleos subtalâmicos. Antigamente relacionados primordialmente à execução motora, esses núcleos são hoje relacionados também a aspectos mais sofisticados do comportamento, como planejamento motor complexo e seqüencial, aprendizagem, direcionamento cognitivo e motivacional. ${ }^{2}$ Essa diversidade de papéis decorre da existência de múltiplos circuitos neuronais fechados, responsáveis pelo processamento de informações a partir do córtex cerebral, passando pelo estriado, pálido e tálamo, e voltando deste ao córtex. ${ }^{3}$ Por cada um desses circuitos corticoestriatais paralelos e segregados, diferentes porções dos GB recebem projeções de áreas específicas do córtex cerebral, ficando em posição estratégica para influenciar e modular o funcionamento do córtex. Cada circuito é responsável pela mediação de funções que correspondem às áreas corticais envolvidas $^{4}$ (Tabela).

Qual a origem da associação entre os gânglios da base e o TOC?

Diversos relatos de caso descrevem a presença de sintomas obsessivo-compulsivos associados a transtornos neurológicos que presumidamente afetam os GB, como: doença de Parkinson, ${ }^{5}$ coréia de Sydenham, ${ }^{6}$ doença de Huntington ${ }^{7}$ e síndrome de Tourette. ${ }^{8}$ Há também relatos de aparecimento de sintomas obsessivo-compulsivos após outras lesões envolvendo os GB,

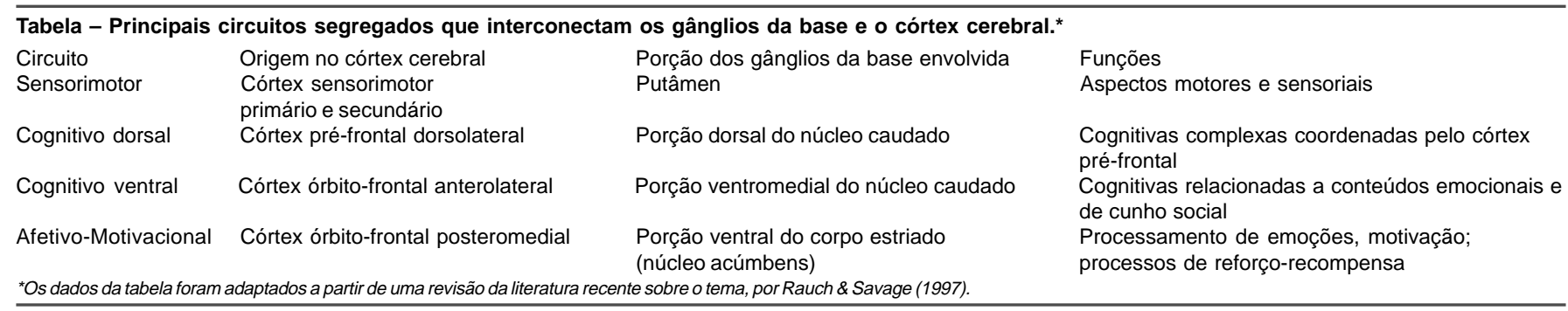


como atrofia após traumatismo cranioencefálico, infartos isquêmicos, ${ }^{9}$ encefalopatias anóxicas e tóxicas. ${ }^{10}$

Outra fonte de suporte à noção de que os GB estão envolvidos na fisiopatologia do TOC vem dos procedimentos cirúrgicos usados em casos refratários a terapêuticas convencionais. Esses tratamentos envolvem invariavelmente a interrupção de fibras do córtex frontal inferior ou giro do cíngulo aos núcleos caudado e putâmen. ${ }^{11}$

Por fim, tem ocorrido nos últimos anos fantástico avanço nos métodos de neuroimagem que permitem a visualização detalhada de aspectos funcionais e estruturais da anatomia cerebral em transtornos neuropsiquiátricos. É essa área de pesquisa, revisada nos próximos itens, que tem permitido uma exploração mais profunda da relação entre alterações dos GB e o TOC.

\section{Neuroimagem do TOC e os gânglios da base}

No TOC, os estudos mais numerosos até hoje têm sido os de neuroimagem funcional, utilizando tomografia por emissão de pósitrons ou fótons (positron emission tomography - PET; single photon computed emission tomography - SPECT) e, mais recentemente, a ressonância magnética (RM) funcional. Estudos de PET e SPECT em repouso demonstram alterações do metabolismo de glicose ou fluxo sangüíneo cerebral regional (FSCr) em caudado-putâmen em pacientes com TOC, comumentemente como aumento de atividade e ocasionalmente como diminuição de FSCr. ${ }^{12}$ Estudos com PET ou RM funcional descrevem também aumento de atividade metabólica nos GB durante a provocação de sintomas em pacientes com TOC. ${ }^{12}$ No entanto, alterações funcionais nos GB não constituem achado universal; há também descrições de atividade normal nessa região cerebral em amostras de pacientes com TOC. ${ }^{13}$

Ainda que menos numerosos, estudos de neuroimagem estrutural têm investigado também alterações volumétricas cerebrais em pacientes com TOC comparados a voluntários normais. Utilizando a tomografia computadorizada (TC), Luxenberg et al $^{14}$ compararam dez pacientes com TOC medicados e dez controles normais e identificaram diminuições no núcleo caudado bilateralmente, mas não em outras regiões cerebrais. No entanto, um estudo subseqüente de Stein et al, ${ }^{15}$ utilizando método semelhante, não replicou esses resultados.

No primeiro estudo volumétrico utilizando a técnica mais sofisticada de RM estrutural, Scarone et al $^{16}$ realizaram avaliações especificamente no núcleo caudado de 20 pacientes com TOC medicados e 16 voluntários normais e identificaram aumento volumétrico à direita no grupo de pacientes. Esses achados contrastam com os resultados de Robinson et al, ${ }^{17}$ que descreveram diminuição do volume do caudado bilateralmente em 26 pacientes com TOC medicados, e com achados negativos de outros estudos. ${ }^{18-21}$

Já Rosemberg et $\mathrm{al}^{22}$ encontraram diminuições volumétricas no corpo estriado em 19 crianças e adolescentes com TOC nunca expostos à medicação psicotrópica, comparados a 19 controles normais.

Apesar de sugerir a existência de alterações estruturais dos GB no TOC, a revisão da literatura acima deixa patentes as controvérsias entre estudos. Além do tamanho modesto das amostras avaliadas e do fator de confusão introduzido pelo uso de medicação, uma outra possível fonte de variabilidade diz respeito aos métodos usados para as medidas volumétricas. Por meio do uso de regiões de interesse (RIs), os GB são delineados manualmente ou de forma semi-automatizada, sempre com dependência da participação do observador. Além disso, é impossível, pelas RI, subdividir os diferentes territórios do caudado e do putâmen.

Nos últimos anos, novos métodos de análise de imagens têm sido desenvolvidos. Um deles é o da morfometria baseada no voxel (MBV), ${ }^{23}$ que permite comparações da concentração de substância cinzenta entre grupos de pacientes e voluntários normais para cada voxel (a unidade tridimensional básica das imagens cerebrais), sem que seja necessário definir a priori as bordas de RI. Numa pesquisa atualmente em andamento, o grupo do presente estudo utiliza a MBV para analisar as imagens de RM estrutural em pacientes com TOC comparados a voluntários normais. Os resultados preliminares mostram, nos pacientes com TOC, diminuição significativa de substância cinzenta localizada num foco na borda entre a porção dorso-medial do núcleo caudado esquerdo e o ventrículo lateral adjacente (Figura A). Entretanto, observam-se também focos de aumento de substância cinzenta em pacientes com TOC numa porção mais ventral e lateral do núcleo caudado e no putâmen à direita (Figura B). Esses resultados preliminares confirmam a importância dos GB na fisiopatologia do TOC e sugerem que é possível identificar alterações distintas em porções diferentes dos GB. Caso confirmados e replicados em outros estudos, esses achados darão suporte à noção de que vários dos circuitos corticoestriatais segregados estão envolvidos na fisiopatologia do TOC. Por exemplo, alterações na porção ventral do núcleo caudado, ao afetar o funcionamento do circuito cognitivo ventral (Tabela), poderiam levar à presença dos sintomas do TOC nas esferas cognitiva e afetiva. ${ }^{24}$ Já lesões localizadas em putâmen poderiam afetar o funcionamento do circuito sensorimotor, levando a fenômenos sensoriais, tiques e compulsões "tiquelike". O uso de métodos cada vez mais sofisticados de neuroimagem será importante para permitir no TOC um

Figura - Representação anatômica de focos de alteração de substância cinzenta nos gânglios da base em pacientes com TOC $(n=20)$ comparados a voluntários normais $(n=13)$, investigada por ressonância magnética estrutural.

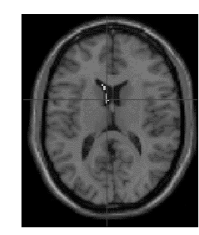

Figura A

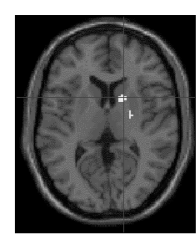

Figura B
Legenda: As imagens mostram (pontos mais claros) focos de alterações volumétricas nos gânglios da base em pacientes com TOC (limiar estatístico de $p<0,001$ ), incluindo: um foco de diminuição de substância cinzenta na borda entre a porção dorsal da cabeça do núcleo caudado esquerdo e o ventrículo lateral adjacente (Figura A) e focos de aumento de substância cinzenta em núcleo caudado ventral e putâmen direito (Figura B). Nesse limiar estatístico, o foco de diminuição de substância cinzenta em caudado dorsal se apresenta com 83 voxels contíguos (de $2 \times 2 \times 2 \mathrm{~mm}$ ), enquanto os focos de aumento de substância cinzenta em caudado ventrale putâmen têm, respectivamente, 45 voxels e 22 voxels. Para facilitar a visualização anatômica, os focos foram superpostos sobre uma imagem de ressonância magnética do cérebro de um sujeito normal, delimitada de acordo com os padrões anatômicos de um atlas estereotáxico. 
mapeamento topográfico de alterações em sub-regiões dos GB e dos circuitos segregados que por elas passam. Se utilizados em amostras amplas e bem caracterizadas clinicamente, esses métodos deverão melhorar o entendimento sobre os mecanismos subjacentes à heterogeneidade de manifestações clínicas desse transtorno.

\section{Os gânglios da base são relevantes para subtipos específicos do TOC?}

Além dos aspectos acima, uma outra possível justificativa para as inconsistências de achados nos estudos de neuroimagem no TOC diz respeito à heterogeneidade clínica desse transtorno. Como as amostras em estudos de neuroimagem são modestas, é plausível que a inclusão de pacientes com subtipos diferentes de TOC leve a variações nos resultados.

Uma das subdivisões do TOC que tem ganhado importância é a dicotomização entre TOC de início precoce e tardio. Aquele ocorre mais freqüentemente em homens, é mais comumente associado a tiques e história familiar de TOC e apresenta resposta a tratamento e prognóstico mais pobres. ${ }^{25}$

A comorbidade entre TOC de início precoce e transtornos de tiques poderia pressupor que alterações nos GB têm papel particularmente relevante nesse subtipo. Em estudos de neuroimagem funcional, a busca dessa associação tem tido resultados pouco promissores. Um estudo de PET revelou aumento de metabolismo no estriado esquerdo em pacientes com TOC de início precoce comparados a controles normais, ${ }^{26}$ mas esses achados não foram replicados num estudo subse- qüente com amostra maior. ${ }^{27}$ Além disso, num estudo recente comparando diretamente os padrões de $\mathrm{FSCr}$ de repouso (com SPECT) entre pacientes com TOC de início precoce e tardio, observaram-se diferenças significativas no tálamo e em córtex frontal inferior, mas não se encontraram alterações no funcionamento dos GB em pacientes com TOC de início precoce. $^{28}$

Estudos de neuroimagem estrutural têm fornecido pistas mais consistentes de que alterações nos GB podem desempenhar papel relevante no TOC de início precoce. Nos estudos nos quais crianças ou adolescentes foram avaliados, os volumes do núcleo caudado e putâmen se mostraram reduzidos nos pacientes com TOC em comparação com controles normais, ${ }^{14,22}$ enquanto volumes normais foram obtidos em pacientes com TOC de início em vida adulta. ${ }^{20}$ No entanto, há dados sugestivos de que mesmo dentre os casos de início precoce deve haver heterogeneidade etiológica quanto ao envolvimento dos GB; em crianças com TOC e/ou transtornos de tiques associados à infecção estreptocócica, estudos de RM estrutural têm apontado aumento (e não diminuição) de volume dos GB, possivelmente refletindo resposta imunológica anormal ao processo infeccioso. ${ }^{29,30}$

Os resultados acima mostram que o estudo de subtipos do TOC por meio da neuroimagem deverá ser mais desenvolvido e poderá contribuir para o esclarecimento das bases etiológicas desse transtorno. Essas investigações poderão ampliar conhecimentos também sobre o envolvimento dos sistemas neurotransmissores no TOC, levando a tratamentos mais eficazes para esse transtorno tão incapacitante.

\section{Referências}

1. Lacerda ALT, Dalgalarrondo P, Camargo EE. Achados de neuroimagem no transtorno obsessivo-compulsivo. Rev Bras Psiquiatr 2001;23:24-7.

2. Mello LEAM, Villares J. Neuronatomy of the basal ganglia. Psychiatr Clin North Am 1997;20:691-704.

3. Alexander GE, Delong MR, Strick PL. Parallel organization of functionally segregated circuits linking basal ganglia and cortex. Annu Rev Neurosci 1986;9:357-81.

4. Rauch SL, Savage CR. Neuroimaging and neuropsychology of the striatum. Bridging basic science and clinical practice. Psychiatr Clin North Am 1997;20:741-68.

5. Daniele A, Bartolomeo P, Casseta E, Bentivoglio AR, Gainotti $\mathrm{G}$, Albanese A, et al. Obsessive-compulsive behaviour and cognitive impairment in a parkinsonian patient after left putaminal lesion. J Neurol Neurosurg Psychiatry 1997;62:288302.

6. Swedo SE, Rapoport JL, Cheslow DL, Leonard HL, Ayoub EM, Hosier DM, et al. High prevalence of obsessive-compulsive symptoms in patients with Sydenham's chorea. Am J Psychiatry 1989;146:246-49.

7. Cummings JL, Cunningham K. Obsessive-compulsive disorder in Huntington's disease. Biol Psychiatry 1992;31:263-70.

8. Saba PR, Dastur K, Keshavan MS, Katerji MA. Obsessivecompulsive disorder, Tourette's syndrome, and basal ganglia pathology on MRI. J Neuropsychiatry Clin Neurosci 1998;10:116-7.
9. Berthier ML, Kulisevsky J, Gironell A, Heras JA. Obsessivecompulsive disorder associated with brain lesions: clinical phenomenology, cognitive function, and anatomic correlates. Neurology 1996;47:353-61.

10. Laplane D, Levasseur M, Pillon B, Dubois B, Baulac M, Mazoyer $\mathrm{B}$, et al. Obsessive-compulsive and other behavioural changes with bilateral basal ganglia lesions. A neuropsychological, magnetic resonance imaging and positron tomography study. Brain 1989;112:699-725.

11. Insel TR, Winslow JT. Neurobiology of obsessive-compulsive disorder. Psychiatry Clin North Am 1992;15:813-24.

12. Saxena S, Brody AL, Schwartz JM, Baxter LR. Neuroimaging and frontal-subcortical circuitry in obsessive-compulsive disorder. $\mathrm{Br}$ J Psychiatry 1998;(Supll 35):26-37.

13. Busatto GF, Zamignani DR, Buchpiguel CA, Garrido GE, Glabus MF, Rocha ET, et al. A voxel-based investigation of regional cerebral blood flow abnormalities in obsessive-compulsive disorder using single photon emission computed tomography (SPECT). Psychiatry Res 2000;99:15-27.

14. Luxenberg JS, Swedo SE, Flament MF, Friedland RP, Rapoport J, Rapoport SI. Neuroanatomical abnormalities in obsessivecompulsive disorder detected with quantitative X-ray computed tomography. Am J Psychiatry 1988;145:1089-93.

15. Stein DJ, Hollander E, Chan S, DeCaria CM, Hilal S, Liebowitz MR, et al. Computed tomography and neurological soft signs in obsessive-compulsive disorder. Psychiatry Res 1993;50:143-50. 
16. Scarone S, Colombo C, Livian S, Abbruzzese M, Ronchi P, Locatelli $\mathrm{M}$, et al. Increased right caudate nucleus size in obsessivecompulsive disorder: detection with magnetic resonance imaging. Psychiatry Res 1992;45:115-21.

17. Robinson D, Wu H, Munne RA, Ashtari M, Alvir JM, Lerner G, et al. Reduced caudate nucleus volume in obsessive-compulsive disorder. Arch Gen Psychiatry 1995;52:393-8.

18. Kellner CH, Jolley RR, Holgate RC, Austin L, Lydiard RB, Laraia $\mathrm{M}$, et al. Brain MRI in obsessive-compulsive disorder. Psychiatry Res 1991;36:45-9.

19. Jenike MA, Breiter HC, Baer L, Kennedy DN, Savage CR, Olivares MJ, et al. Cerebral structural abnormalities in obsessive-compulsive disorder. A quantitative morphometric magnetic resonance imaging study. Arch Gen Psychiatry 1996;53:625-32.

20. Aylward EH, Harris GJ, Hoehn-Saric R, Barta PE, Machlin SR, Pearlson GD. Normal caudate nucleus in obsessive-compulsive disorder assessed by quantitative neuroimaging. Arch Gen Psychiatry 1996;53:577-84.

21. Stein DJ, Coetzer R, Lee M, Davids B, Bouwer C. Magnetic resonance brain imaging in women with obsessive-compulsive disorder and trichotillomania. Psychiatry Res 1997;74:177-82.

22. Rosenberg DR, Keshavan MS, O'Hearn KM, Dick EL, Bagwell WW, Seymour AB, et al. Frontostriatal measurement in treatment-naive children with obsessive-compulsive disorder. Arch Gen Psychiatry 1997;54:824-30.

23. Ashburner J, Friston KJ. Voxel-based morphometry - the methods. Neuroimage, 2000;11:805-21.
24. Eichstedt JA, Arnold SL. Childhood-onset obsessive-compulsive disorder: a tic-related subtype of OCD? Clin Psychol Rev 2001;21:137-58

25. Ackerman DL, Greenland S, Bystritsky A, Morgenstern H, Katz RJ. Predictors of treatment response in obsessive-compulsive disorder: Multivariates analyses from a multicenter trial of clomipramine. J Clin Psychopharmacol 1994;14:247-54.

26. Swedo SE, Schapiro MB, Grady CL, Cheslow DL, Leonard HL, Kumar A, et al. Cerebral glucose metabolism in childhood-onset obsessive compulsive disorder. Arch Gen Psychiatry 1989;46:518-23.

27. Benkelfat C, Nordahl TE, Semple WE, King AC, Murphy DL, Cohen RM. Local cerebral glucose metabolic rates in obsessivecompulsive disorder. Arch Gen Psychiatry 1990;47:840-8.

28. Busatto GF, Buchpiguel CA, Zamignani DR, Garrido GE, Glabus MF, Rosario-Campos MC, et al. Regional cerebral blood flow abnormalities in early-onset obsessive-compulsive disorder: an exploratory SPECT study. J Am Acad Child Adolesc Psychiatry 2001;40:347-54.

29. Giedd JN, Rapoport JL, Garvey MA, Perimutter S, Swedo SE. MRI Assessment of children with obsessive-compulsive disorder or tics associated with streptococal infection. Am J Psychiatry 2000;157:281-3.

30. Peterson BS, Leckman JF, Tucker D, Scahill L, Staib L, Zhang H, et al. Preliminary findings of antistreptococcal antibody titers and basal ganglia volumes in tic, obsessive-compulsive, and attention deficit/hyperactivity disorders. Arch Gen Psychiatry 2000;57:364-72

Correspondência: Geraldo Busatto Filho

Departamento de Psiquiatria da Faculdade de Medicina da USP - Rua Dr. Ovídio Pires de Campos, s/nº - 05403-010 São Paulo, SP, Brasil Tel.: (0xx11) 3081-5411 -E-mail: geraldo.busatto@ honet.usp.br 